\title{
A Summary of the Malaysian Clinical Practice Guidelines on Management of Obesity 2004
}

\author{
Suehazlyn Zainudin ${ }^{1}$, Zaiton Daud ${ }^{2}$, Masni Mohamad ${ }^{3}$, \\ Alexander Tan Tong Boon ${ }^{4}$, Wan Mohd Izani Wan Mohamed ${ }^{5}$ \\ ${ }^{1}$ Faculty of Medicine, National University of Malaysia, \\ ${ }^{2}$ Nutrition Division, Ministry of Health \\ Department of Medicine, Hospital Putrajaya, Malaysia \\ ${ }^{4}$ Faculty of Medicine, University of Malaya Medical Centre \\ ${ }^{5}$ Faculty of Medicine, Hospital University Sains Malaysia
}

\begin{abstract}
In 2004, the Malaysian Clinical Practice Guideline for Management of Obesity was published to assist health practitioners in the management of the obese population. This guidelines, based on gathered evidence published up to 2004, defined weight classifications according to BMI suited to the Malaysian population. The guideline also discussed methods of assessing obesity in adults. Recommendations with respect to treatment approach, encompassing lifestyle, dietary, pharmacological and surgical management of obesity in adults, and management approach in children and adolescent were also discussed in detail in the guidelines. The following article summarizes the recommendations that were made in the 2004 guidelines.
\end{abstract}

Keywords: clinical practice guidelines, obesity management, Malaysia

\section{Introduction}

In 1996, the National Health and Morbidity Survey in Malaysia reported a prevalence of $15.1 \%$ overweight and $2.9 \%$ obesity among adult males, whilst in adult females, $17.9 \%$ were overweight and $5.7 \%$ obese ${ }^{1}$. The prevalences did not differ much between rural and urban population, and there were more obese Malays and Indians, compared to Chinese.

These guidelines were developed with the objective of assisting healthcare providers to better diagnose and manage overweight and obese patients. It was a combined initiative of the Malaysian Association for the Study of Obesity (MASO) and the Malaysian Endocrine and Metabolic Society (MEMS). It recommends a multidisciplinary approach in the management of overweight and obese patients in Malaysia.

The evidence from this guideline was collated from several sources, including systematic review of relevant published literature (up to 2004) as identified by electronic search, and reports of relevant expert working groups ${ }^{2-7}$.

There are 6 main sections in the guidelines; Section1Introduction, Section 2- Diagnosis and Assessment of Obesity in Adults, Section 3- Therapy: Overall Approach, Section 4-Therapy: Lifestyle Advice, Section 5- Therapy:

ISSN 0857-1074

Copyright $\odot 2011$ by the JAFES

Received October 11, 2011. Accepted October 11, 2011.
Pharmacotherapy and Surgical Therapy and Section 6Childhood and Adolescent Obesity. Section 7 consists of appendices including behavioural assessment, sample menu plan, food groups and exchange lists, determination of activity status for calculating calorie requirements for weight reduction and maintenance, surgical inventions in obesity and the IOTF cut-off points of BMI for overweight and obesity by sex from 2-18 years, as well as body mass index-for-age percentiles for boys and girls: CDC growth charts: United States.

\section{Definition of Obesity}

Obesity is a complex multifactorial condition characterised by excess fat. Generally, men with $>25 \%$ body fat and women with $>35 \%$ body fat are considered obese. The body mass index (BMI), which is defined as Weight $(\mathrm{kg}) /$ Height ${ }^{2}\left(\mathrm{~m}^{2}\right)$, is the most widely established and widely used measurement. Evidence from several Asian countries showed that the risk of comorbidities begin to rise at a lower BMI value than that defined by $\mathrm{WHO}$ as overweight and obese ${ }^{8-14 h}$. At a similar BMI, many Asian populations have a higher body fat content as compared with Caucasian/European population ${ }^{15-18}$.

Based on this, the guideline recommends the classification of weight by BMI, according to the risk of comorbidities as stated in Table 1.
Corresponding author: Suehazlyn Zainudin MD, MMed

Division of Diabetes \& Endocrinology,

National University of Malaysia Medical Centre,

Jalan Yaacob Latif, Bandar Tun Razak, Cheras,

56000 Kuala Lumpur, Malaysia.

Tel: +603-91456958

E-Mail: suehazlyn@gmail.com 
The guidelines also recommended that waist circumference (WC) is used to assess abdominal fat content as it is simple and reliable. WC measurements correlates well with abdominal fat content irrespective of $\mathrm{BMI}$ and is a independent risk factor for cardiovascular diseases. It is most useful in patients with normal or overweight BMI but is deemed unnecessary in patients with $\mathrm{BMI}>35 \mathrm{~kg} / \mathrm{m}^{2}$, as it loses its predictive value.

Current evidence suggests that among Asians, WC of 90 $\mathrm{cm}$ in men and $80 \mathrm{~cm}$ in women is associated with increased risk of comorbidities ${ }^{8-11}$.

\begin{tabular}{lll}
\multicolumn{3}{c}{ Table 1. Classification of weight by BMI } \\
\hline \multicolumn{1}{c}{ Classification } & \multicolumn{1}{c}{ BMI (kg/m2) } & \multicolumn{1}{c}{ Risk of Comorbidities } \\
\hline Underweight & $<18.5$ & $\begin{array}{l}\text { Low (but increase risk of other } \\
\text { clinical problems) } \\
\text { Increasing but acceptable risk }\end{array}$ \\
Normal range & $18.5-22.9$ & $\begin{array}{l}\text { Increased } \\
\text { Overweight }\end{array}$ \\
$\begin{array}{l}\geq 23 \\
\text { Pre-obese }\end{array}$ & $23.0-27.5$ & High \\
Obese I & $27.5-34.9$ & Very High \\
Obese II & $35.0-39.9$ & Extremely High \\
Obese III & $\geq 40$ &
\end{tabular}

Excessive weight results in an increase overall mortality and morbidity ${ }^{19}$. The risk of cardiovascular diseases and its risk factors is greatest in patients with abdominal obesity. The relative risk of developing type 2 diabetes, gallstones, fatty liver, dyslipidaemia, metabolic syndrome, breathlessness and sleep apnoea is increased 3-fold. Other increased health risks include coronary artery disease, cerebrovascular disease, cardiac failure, hypertension, hyperuricaemia, cancer (breast, endometrium, colorectal, liver, prostate, gallbladder), polycystic ovarian syndrome, reproductive hormone abnormalities and impaired fertility, low back pain and foetal defects associated with maternal obesity. Weight loss has advantages in reducing these risk factors and the risk of obesity related diseases.

\section{Diagnosis and Assessment of Obesity in Adults}

Obesity in adults can be diagnosed by performing a comprehensive medical evaluation which includes patient's family history, physical examination and laboratory tests. An assessment of patient's eating habits including snacking, his/her habitual physical activity (frequency, type, duration) and a psychological status evaluation (body image, eating disorders) should be performed. Additionally, physical examination should include assessment of degree of obesity and body fat distribution with special attention to potential comorbidities. The laboratory tests that should be done are fasting blood glucose, fasting blood lipid profile and biochemistry profile. If indicated, thyroid function should be checked, as well as 24-hour urine free cortisol and investigations to exclude polycystic ovarian syndrome should be carried out.

The current diagnostic criteria of overweight and obesity for adults are set at above $23 \mathrm{~kg} / \mathrm{m}^{2}$ and $27.5 \mathrm{~kg} / \mathrm{m}^{2}$, respectively. In addition to BMI, waist and hip circumferences are also measured for monitoring the abdominal fat distribution and reference value for waisthip ratio for men and women are 0.90 and 0.85 , respectively. Even though BMI is the most established measurement used to monitor the progress of an individual's weight, there are some limitations in estimating the degree of obesity under certain circumstances which include overestimations in very muscular individuals and underestimations in individual who have lost muscle mass.

Obesity-associated diseases which will contribute to added cardiovascular morbidity and mortality for eg, coronary heart disease and cardiovascular risk factors, should be identified. They provide an additional guide to the need and intensity of weight reducing intervention. Identification of other related diseases which are increased amongst obese patients such as osteoarthritis, gallstones and stress incontinence are also crucial in planning for appropriate weight management strategies.

Obesity is primarily caused by the interaction between genetic predisposition and environmental factors. These include social and behaviour factors, sedentary lifestyle, iatrogenic causes, endocrine obesities and genetic obesities. These should be identified and managed appropriately.

\section{Therapy: Overall Approach}

The general goals for obesity therapy are to achieve weight loss, maintain lower body weight, prevent further weight gain and to treat the comorbidities/underlying causes. The initial goal of weight loss is to reduce approximately $10 \%$ body weight from baseline. A calorie deficit of 500 to1000 $\mathrm{kcal} /$ day can result in weight loss at a rate of 0.5 to 1 $\mathrm{kg} /$ week. The combined modalities of therapy (diet, physical activity and behavior therapy) must be continued indefinitely to ensure weight maintenance. These are best done in the form of a multidisciplinary team approach, involving physicians interested in management of obesity or endocrinologists, dieticians, physiotherapists, psychologists or psychiatrists, and when indicated, the bariatric surgeon.

The recommended treatment options for the treatment of overweight and obesity in adults depends on different levels of BMI and other risk factors. Lifestyle modifications including dietary intervention, physical activity and behavior therapy is the mainstay of treatment for all levels of BMI. Anti-obesity drugs should be considered at BMI of $27.5 \mathrm{~kg} / \mathrm{m} 2$ and above, and BMI 25.0-27.4 kg/m2 with presence of more than 2 risk factors. Very low calorie diet as a treatment option can be considered at BMI of 27.5-34.9 $\mathrm{kg} / \mathrm{m} 2$ with $\geq 2$ risk factors and $\mathrm{BMI}>35.0 \mathrm{~kg} / \mathrm{m} 2$ with or without risk factors. Surgery is suggested at BMI of 35.0 to $39.9 \mathrm{~kg} / \mathrm{m} 2$ with $\geq 2$ risk factors and BMI $\geq 40.0$ with or without risk factors. Regular and frequent consultations with the multidisciplinary or obesity team, initially weekly 
to biweekly; subsequently monthly for at least a year, is beneficial and a major determinant of successful weight loss and control.

\section{Lifestyle Advice}

Lifestyle advice encompasses dietary therapy, physical activity and behavior therapy. Dietary intervention aims to reduce weight through a decrease in calories consumption. Diet strategies include low calorie diet (LCD), lower-fat diet, very low-calorie diet (VLCD), highprotein low carbohydrate diet and dietary education. LCD is where calorie deficit of 500 to $1000 \mathrm{kcal} /$ day from maintenance requirement for weight loss and prevention of weight regain. Reducing fat as part of a low calorie diet is a practical way to reduce calories. Very Low Calorie Diet (VLCD) where calorie restriction between 200 to 800 $\mathrm{kcal} /$ day may be indicated for moderately to severely obese patients (BMI $>30 \mathrm{~kg} / \mathrm{m}^{2}$ ). It can result in quick, short-term weight losses, but medical supervision is required. All weight management strategies should include education in healthy eating habits.

Physical activity should be an integral part of weight loss therapy and weight maintenance because it contributes to weight loss, decreases abdominal fat and increases cardiorespiratory fitness. Individuals require $45-60$ minutes per day of moderate intensity activity or lesser amounts of vigorous intensity activity. Weight loss is more likely to be achieved and maintained by behavioural modification techniques focusing on lifestyle and attitude. The goal of behaviour therapy is to alter the eating habits of the obese individual.

\section{Pharmacotherapy}

Pharmacotherapy is an adjunct to lifestyle changes in some patients with BMI $>27.5 \mathrm{~kg} / \mathrm{m}^{2}$ or BMI $>25 \mathrm{~kg} / \mathrm{m}^{2}$ with comorbidities. Drugs should only be used under careful medical supervision and as part of a long-term treatment strategy. The benefit of pharmacotherapy must be carefully weighed against risks of side effects.

Orlistat: A peripherally acting pancreatic lipase inhibitor. Dose is $120 \mathrm{mg}$ tds with each main meal containing fat (during or up to 1 hour after meal); omit if meal is missed or does not contain fat. Side effects include loose stools and malabsorption of fat soluble vitamins. Contraindications are chronic malabsorption syndrome and cholelithiasis.

Phentermine: An amphetamine derivative that should only be used for $<3$ months. Dose is $8 \mathrm{mg} \mathrm{tds}, 30 \mathrm{~min}$ before meals or $15-30 \mathrm{mg} /$ day before breakfast or $10-14 \mathrm{hrs}$ before bed. Side effects include increase in blood pressure, insomnia and nervousness. Contraindications are pulmonary hypertension, heart valve abnormalities, moderate to severe hypertension, cerebrovascular disease, severe cardiac disease, hypersensitivity, hyperthyroidism, psychiatric illness, glaucoma and drug/alcohol dependence.

\section{Other drugs that favour weight loss:}

Fluoxetine: Anti-depressant and appetite suppressant. Not specifically approved for weight loss. Starting dose is $20 \mathrm{mg} /$ day in the morning, may increase by $20 \mathrm{mg} /$ day each week to maximum of $60 \mathrm{mg} /$ day. Side effects include anxiety, drowsiness, insomnia and nervousness. Contraindications are hypersensitivity and concomitant use of monoamine oxidase inhibitors (MAOIs).

Metformin: Not specifically licensed for obesity but may be useful for managing obesity in patients with dysglycaemia or PCOS. Dose is 500 to $1000 \mathrm{mg} \mathrm{bd} / \mathrm{tid}$ with meals. Side effects include nausea, flatulence, bloating, diarrhea and rarely lactic acidosis. Contraindications include hypersensitivity, diabetic ketoacidosis, renal dysfunction and acute conditions that may predispose to renal dysfunction (e.g. infection, contrast agents), acute or chronic disease that may cause tissue hypoxia (e.g. cardiac or respiratory failure) and lactation.

Pharmacotherapy is contraindicated in children, pregnant and lactating women and patients who have previously suffered adverse effects from drugs in this category. Combination pharmacotherapy cannot be recommended.

\section{Surgery for Weight Loss}

Surgical intervention for obesity can result in substantial and sustained weight loss, but should be reserved for patients above 18 years of age with morbid obesity (BMI > $40 \mathrm{~kg} / \mathrm{m}^{2}$ ) or BMI of $35-40 \mathrm{~kg} / \mathrm{m}^{2}$ with weight-related comorbidities and have failed other therapies provided by experts in obesity management. In addition, the patient has to be motivated and has no contraindications for anaesthesia and surgery. The common surgical procedures are; gastric banding, gastric bypass (Roux-en-Y) and the biliopancreatic bypass surgery. Complications of surgery include malabsorption, "dumping syndrome," gallstones disease and depression. Lifelong medical and nutrition surveillance after surgical therapy should include monitoring of indices of inadequate nutrition and modification of any preoperative disorders.

\section{Childhood and Adolescent Obesity}

Obesity among children and adolescents are commonly due to unhealthy eating patterns and lack of physical activity. Occasionally, it is due to genetic factors or endocrine disorders. It is arbitrarily defined as a BMI greater than the 95th percentile. Childhood obesity is associated with an increase in prevalence of Type 2 diabetes among young. Factors which determine persistence of obesity into adulthood are; onset of obesity after the age of three, degree of obesity and presence of obesity in at least one of the parents. 
Management of children and adolescent with obesity is aimed at reducing the amount of body fat. Growing children often do not need to lose weight, however older adolescents who have attained their final height should make efforts to lose excess weight by dietary modification and increase in physical activities. It is recommended to aim for weight loss of $2-4 \mathrm{~kg}$ initially, or at a rate of 0.5 to 2 $\mathrm{kg}$ per month. This requires continuous good support and commitment from the whole family members.

\section{Conclusion}

In the treatment of obesity, the need for multidisciplinary approach cannot be overemphasized. Treatment strategies should be tailored according to the individual needs. The Malaysian Clinical Practice Guideline for Obesity was developed with the hope that it will help strengthen the management of obesity in the country. The full downloaded document (http://www.acadmed.org.my/) is made readily available online for easy reference.

\section{Acknowledgements}

Members of the CPG panel: Prof. Dr. Ikram Shah, Prof. Dr. Wan Mohamed Wan Bebakar, Prof. Mohd Ismail Noor, Prof. Dr. Nor Azmi Kamaruddin, Prof. Rabindarjeet Singh, Datuk Dr. Noor Hisham Abdullah, Dr. Zanariah Hussein, Dr. Fuziah Md Zain, Ms Lee Lai Fun, Ms Siti Hawa Mohd Taib.

\section{References}

1. Lim TO, Ding LM, Zaki M et al. Distribution of body weight, height and Body Mass Index in a national sample of Malaysian adults. The Medical Journal of Malaysia. 2000;55(1):108-28.

2. Scottish Intercollegiate Guidelines Network. Obesity in Scotland: Integrating prevention with weight management. Edinburgh: Scottish Intercollegiate Guidelines Network; 1996.

3. National Institutes of Health. Clinical guidelines on the identification, evaluation, and treatment of overweight and obesity in adults: The evidence report. Report No: 98-4083. Bethesda: National Institutes of Health; September 1998.

4. World Health Organization, International Obesity Task Force, International Association for the Study of Obesity. The Asia-Pacific perspective: Redefining obesity and its treatment. Hong Kong: WHO, IOTF \& IASO; 2000.

5. World Health Organization. Obesity: Preventing and managing the global epidemic. Report on a WHO Consultation on Obesity, Geneva, June 1997. Geneva: World Health Organization; 1998.

6. AACE/ACE Obesity Task Force. AACE/ACE Position statement on the prevention, diagnosis and treatment of obesity. Endocrine Practice. 1998;4(5):297-350.

7. WHO Expert Consultation. Appropriate Body Mass Index for Asian populations and its implications for policy and intervention strategies. Lancet. 2004;363:157-163.

8. Deurenberg P. Universal cut-off BMI points for obesity are not appropriate. Br J Nutr. 2001;85:135-6.

9. Cooperative Meta-Analysis Group of China Obesity Task Force. Predictive value of Body Mass Index and waist circumference to risk factors of related diseases in Chinese adult population. Clinical Journal of Epidemiology. 2002;23:5-10.

10. Zhou BF. Predictive values of Body Mass Index and waist circumference for risk factors of certain related diseases in Chinese adults-study on optimal cut-off points of body mass index and waist circumference in Chinese adults. Biomedical and Environmental Sciences. 2002;15:83-95.

11. Jia WP, Xiang KS, Chen L et al. Epidemiological study on obesity and its comorbidities in urban Chinese older than 20 years of age in Shanghai, China. Obesity Reviews. 2002;3(3):157-65.
12. Yajnik CS. The lifecycle effects of nutrition and body size on adult adiposity, diabetes and cardiovascular disease. Obesity Reviews. 2002;3(3):217-24.

13. Reddy KS, Prabhakaran D, Shah P, Shah B. Differences in Body Mass Index and waist: hip ratios in North Indian rural and urban populations. Obesity Reviews. 2002;3(3):197-202.

14. Yoshiike N, Seino F, Tajima $\mathrm{S}$ et al. Twenty-year changes in the prevalence of overweight in Japanese adults: The National Nutrition Survey 1976-95. Obesity Reviews. 2002;3(3):183-90.

15. Wang J, Thornton JC, Russell M et al. Asians have lower BMI (BMI) but higher percent body fat than do Whites: Comparisons of anthropometric measurements. Am J Clin Nutr. 1994;60:23-8.

16. Deurenberg-Yap M, Schmidt G, Staveren WA, Deurenberg P. The paradox of low Body Mass Index and high body fat percent among Chinese, Malays and Indians in Singapore. Intl J Obesity. 2000;24:1011-7.

17. He M, Tan KCB, Li ETS, Kung AWC. Body fat determinations by dual energy X-ray absorptiometry and its relation to Body Mass Index and waist circumference in Hong Kong Chinese. Intl J Obesity. 2001;25:748-52.

18. Gurrici S, Hartriyanti Y, Hautvast JG, Deurenberg P. Relationship between body fat and Body Mass Index: Differences between Indonesians and Dutch Caucasians. Eur J Clin Nutr. 1998;52(11):77983.

19. Manson JE, Willett WC, Stampfer MJ et al. Body weight and mortality among women. N Engl J Med. 1995;333(11):677-85. 